\title{
An Optimal Algorithm for Detecting Pseudo-squares ${ }^{\star}$
}

\author{
Srečko Brlek and Xavier Provençal \\ Laboratoire de Combinatoire et d'Informatique Mathématique, \\ Université du Québec à Montréal, \\ CP 8888, Succ. Centre-ville, Montréal (QC) Canada H3C3P8 \\ \{brlek, provenca\}@lacim.uqam.ca
}

\begin{abstract}
We consider the problem of determining if a given word, which encodes the boundary of a discrete figure, tiles the plane by translation. These words have been characterized by the Beauquier-Nivat condition, for which we provide a linear time algorithm in the case of pseudo-square polyominoes, improving the previous quadratic algorithm of Gambini and Vuillon.
\end{abstract}

\section{Introduction}

In discrete geometry many results are based on an arithmetic approach for characterizing and recognizing patterns having a certain shape. Here we take a combinatorics on words point of view that enable us with new tools for analyzing a shape in discrete planes.

The problem of deciding if a given polyomino tiles the plane by translation goes back to Wisjhoff and Van Leeuven 12 who coined the term exact polyomino for these, and also provided a polynomial $\mathcal{O}\left(n^{4}\right)$ algorithm for solving the problem. Polyominoes may be coded by words on a 4 -letter alphabet $\Sigma=\{a, \bar{a}, b, \bar{b}\}$, also known as the Freeman chain codes [5,6] coding their boundaries (see [1] for further reading). For instance, the boundary $\mathbf{b}(P)$ of the polyomino in Figure 1 (a), in a counterclockwise manner, is coded by the word

(a):

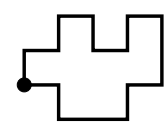

(b):

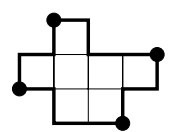

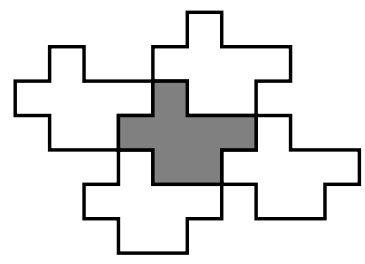

Fig. 1. (a) a polyomino; (b) an exact polyomino

$w=a \bar{b} a a b a b b \bar{a} \bar{b} \bar{a} b \bar{a} \bar{b} \bar{a} \bar{b}$. Observe that we may consider the words as circular which avoids to fix an origin. The perimeter of a polyomino $P$ is the length of

\footnotetext{
* With the support of NSERC (Canada).
} 
its boundary word $\mathbf{b}(P)$ and is of even length $2 n$. Beauquier and Nivat 2] gave a characterization stating that the boundary of such a polyomino $P$ may be factorized (not necessarily in a unique way) as

$$
\mathbf{b}(P)=A \cdot B \cdot C \cdot \widehat{A} \cdot \widehat{B} \cdot \widehat{C}
$$

where at most one of the variables is possibly empty, and where $\widehat{()}$ is defined by $\widehat{U}=\widetilde{\bar{U}}, \widetilde{()}$ being the usual reversing operation and $\overline{(~)}$ the transformation on $\Sigma=\{a, \bar{a}, b, \bar{b}\}$ sending each letter $\alpha \in \Sigma$ on its complement $\bar{\alpha}$. For instance, the exact polyomino in Figure 1 (b) is coded by the circular word

$$
w=a a b a b \bar{a} \bar{a} b \bar{a} \bar{b} \bar{a} \bar{b} a \bar{b},
$$

its semi-perimeter is 7 , and its boundary may be factorized as

$$
\mathbf{b}(P)=A \cdot B \cdot \widehat{A} \cdot \widehat{B}=a \bar{b} a a \cdot b a b \cdot \bar{a} \bar{a} b \bar{a} \cdot \bar{b} \bar{a} \bar{b} .
$$

Determining if a given word $w \in \Sigma^{n}$ is the boundary of a polyomino is computed in $\mathcal{O}(n)$. Therefore the problem reduces to find a factorization satisfying the Beauquier and Nivat condition. Recently, Gambini and Vuillon 7 improved the Wisjhoof-van Leeuven bound by designing an $\mathcal{O}\left(n^{2}\right)$ algorithm that checks the Beauquier-Nivat condition [1.

Our algorithms borrow from Lothaire 11] that the Longest-Common-Factor, the Longest-Common-Prefix and the Longest-Common-Suffix in two words may be computed in linear time. The approach is also inspired by the linear algorithm of Gusfield and Stoye [8] for detecting tandem repeats in a word, and by the linear algorithm used to detect repetitions with gaps, as shown in Lothaire 11. More precisely, the computation of the Longest-Common-Left-Extension (LCLE) and Longest-Common-Right-Extension (LCRE) is achieved in constant time, provided a linear preprocessing is performed on $u$ and $v$, by a clever utilization of suffix trees (see Gusfield [9]). Taking advantage of these algorithms we provide a linear algorithm, with respect to the length of words, for pseudo-square polyominoes.

\section{Preliminaries}

Let $\Sigma$ be a finite alphabet whose elements are called letters. Finite words are sequences of letters, that is, functions $w:[0 . . n-1] \longrightarrow \Sigma$, and the set of words of length $n$ is denoted $\Sigma^{n}$. The free monoid $\Sigma^{*}=\cup_{n=0}^{\infty} \Sigma^{n}$ is the set of all finite words and the empty word is denoted $\epsilon$.

A morphism is a function $\sigma: \Sigma^{*} \longrightarrow \Sigma^{*}$ such that $\sigma(u v)=\sigma(u) \sigma(v)$. Clearly a morphism is defined by the image of the letters. A factor $f$ of $w$ is a word $f \in \Sigma^{*}$ satisfying

$$
\exists x \in \Sigma^{*}, y \in \Sigma^{*}, w=x f y .
$$

If $x=\epsilon$ (resp. $y=\epsilon$ ) then $f$ is called prefix (resp. suffix). The set of all factors of $w$ is denoted by $F(w)$, and those of length $n$ is $F_{n}(w)=F(w) \cap \Sigma^{n}$. Finally 
$\operatorname{Pref}(w)$ denotes the set of all prefixes of $w$. The length of a word $w$ is $|w|$, and the number of occurrences of a factor $f \in \Sigma^{*}$ is $|w|_{f}$. A word is said to be primitive if it is not a power of another word. If $w=p u$, and $|w|=n,|p|=k$, then $p^{-1} w=w[k+1] . . w[n-1]=u$ is the word obtained by erasing $p$. As a special case when $|p|=1$ we have the shift operator $\sigma$ defined by $\sigma(w)=$ $w[1 . .(n-1)]$. Another useful operator is the circular permutation $\rho$ defined by $\rho(w)=w[1 . .(n-1)] \cdot w[0]$.

Two words $u$ and $v$ are conjugate when there are words $x, y$ such that $u=x y$ and $v=y x$. Equivalently, $u$ and $v$ are conjugate if and only if there exists an index $k$ such that $u=\rho^{k}(v)$. Conjugaison is an equivalence relation written $u \equiv$ $v$. The mirror image $\widetilde{u}$ of $u=u_{1} u_{2} \cdots u_{n} \in \Sigma^{n}$ is the word $\widetilde{u}=u_{n} u_{n-1} \cdots u_{1}$. A palindrome is a word $p$ such that $p=\widetilde{p}$, and for a language $L \subseteq \Sigma^{\infty}$, we denote by $\operatorname{Pal}(L)$ the set of its palindromic factors.

Paths on the square lattice $\mathbb{Z} \times \mathbb{Z}$ are encoded on the alphabet $\Sigma=\{a, \bar{a}, b, \bar{b}\}$ identified with the unit steps $\{\rightarrow, \leftarrow, \uparrow, \downarrow\}$. Parallel paths always define a translation and we say that two words are homologue when the corresponding paths define a translation. More precisely, two words $u$ and $v$ are said homologue when either

(i) $u=v$, or

(ii) $u=\widehat{v}$.

An exact polyomino $P$ whose boundary is $\mathbf{b}(P)=A \cdot B \cdot C \cdot \widehat{A} \cdot \widehat{B} \cdot \widehat{C}$ is called a pseudo-hexagon if none of the variables is empty and a pseudo-square otherwise. In this factorization $A$ (resp. $B, C$ ) and $\widehat{A}$ (resp. $\widehat{B}, \widehat{C}$ ) are homologue and define the respective translations. For instance, the translations defined by the homologue sides of the pseudo-square polyomino

$$
\mathbf{b}(P)=A \cdot B \cdot \widehat{A} \cdot \widehat{B}=a \bar{b} a a \cdot b a b \cdot \bar{a} \bar{a} b \bar{a} \cdot \bar{b} \bar{a} \bar{b}
$$

are shown in Figure 2 (a). In the case of a pseudo-hexagon, as in Figure 2(b),

(a):

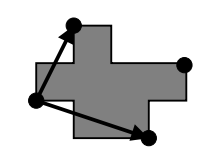

(b):

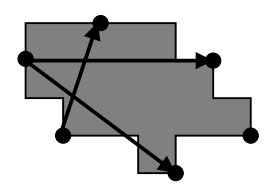

Fig. 2. Translations defined by homologue sides of a polyomino tile

the translations are related by the relation $t_{3}=t_{1}+t_{2}$. Moreover, the relative positions of the starting and ending point of any path is completely determined by the sum of the unit vectors corresponding to each letter. By abuse of notation we write for a path $w:[0 . . n-1] \rightarrow \Sigma$

$$
\vec{w}=\sum_{k=0}^{n-1} \overrightarrow{w_{k}} .
$$

Note that $\vec{w}=0$ if and only if $w$ is a closed path, and that $\vec{u}=-\overrightarrow{\widehat{u}}$. 


\section{Searching the Homologue Factors}

Since polyominoes are coded by circular words $w$, in order to find the homologue factors it is convenient to work with $w \cdot w$ since a pair of homologue factors might be split, depending on the starting point.

Therefore, finding the homologue factors amounts to look for the longest common factor of $w w$ and $\widehat{w w}$ denoted $\operatorname{LCF}(w w, \widehat{w w})$.

For instance the longest common factors of the polyomino-tile $P$ in Figure 1 (b) are

$$
\mathrm{LCF}(w w, \widehat{w w})=\{a \bar{b} a a, \bar{a} \bar{a} b \bar{a}\}
$$

and they are necessarily homologue sides(!). Indeed, since we know the positions $i$ and $j$ of $a \bar{b} a a$ and $\bar{a} \bar{a} b \bar{a}$ in $w$, this is easy to check in linear time. Clearly the boundary of $P$ may be written as

$$
\mathbf{b}(P)=w=a \bar{b} a a \cdot u \cdot \bar{a} \bar{a} b \bar{a} \cdot v
$$

and then one easily checks that $v=\widehat{u}$. Unfortunately the situation is not always that good. Indeed, let $w=a a b b b a a b \bar{a} \bar{a} b \bar{a} \bar{b} \bar{b} \bar{b} \bar{a} \bar{a} \bar{b} a \bar{b}$. Then the longest homologue factors of $w$ are (see Figure 3)

$$
\operatorname{LCF}(w w, \widehat{w w})=\{a a b b b a, \bar{a} \bar{b} \bar{b} \bar{b} \bar{a} \bar{a}\},
$$

but $w=a a b b b a \cdot a b \bar{a} \bar{a} b \cdot \bar{a} \bar{b} \bar{b} \bar{b} \bar{a} \bar{a} \cdot \bar{b} a \bar{b}$ does not satisfy the Beauquier-Nivat condition. A good factorization is $w \equiv b b \cdot b a a b \cdot \bar{a} \bar{a} b \bar{a} \cdot \bar{b} \bar{b} \cdot \bar{b} \bar{a} \bar{a} \bar{b} \cdot a \bar{b} a a$.

(a):

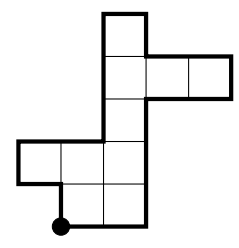

(b):

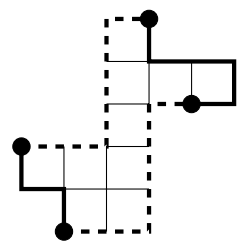

(c):

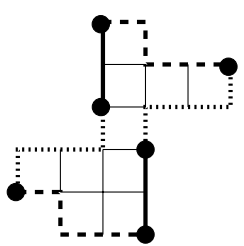

Fig. 3. (b) longest homologue factors; (c) a good factorization

This means that not all the homologue factors provide a factorization, and good candidates are those separated by factors of same length.

Definition 1. Let $w \equiv \mathbf{b}(P)$ be the boundary word of a polyomino $P$. A factor $A$ of $w$ is admissible if

(i) $w \equiv A x \widehat{A} y$, for some $x, y$ such that $|x|=|y|$;

(ii) $A$ is saturated, that is, $x_{0} \neq \overline{x_{k-1}}$ and $y_{0} \neq \overline{y_{k-1}}$ where $k=|x|=|y|$.

Nevertheless, admissibility is ensured for words that code the boundary of polyominoes. Indeed, Gambini and Vuillon established the following property ( 7], section 3.1) by using a geometric result of Daurat and Nivat 4]. 
Lemma 1. Let $w \equiv A B C \widehat{A} \widehat{B} \widehat{C}$ be a Beauquier-Nivat factorization of the boundary $\mathbf{b}(P)$ of an exact polyomino $P$. Then $A, B$ and $C$ are admissible.

Conversely, not all admissible factors lead to a Beauquier-Nivat factorization. For instance, in the polyomino $w \equiv$ a a $a b a b \bar{a} b \bar{a} \bar{a} \bar{a} \bar{b} \bar{a} \bar{b} a \bar{b}$ shown below, the

(a):

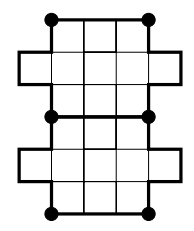

(b):

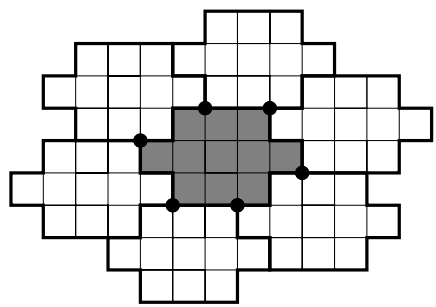

factor $a a a$ is admissible but does not provide a correct factorization of $w$. Indeed, $A=a a$ is the admissible factor $(w=A x \widehat{A} y$ with $x=a b a b \bar{a} b, y=\bar{a} \bar{b} \bar{a} \bar{b} a \bar{b})$ yielding a correct factorization with $B=a b a$ and $C=b \bar{a} b$ :

$$
w \equiv a a \cdot a b a \cdot b \bar{a} b \cdot \bar{a} \bar{a} \cdot \bar{a} \bar{b} \bar{a} \cdot \bar{b} a \bar{b} .
$$

The following proposition establishes a useful property.

Proposition 1. Let $w=\mathbf{b}(P) \in \Sigma^{2 n}$ be the contour of a polyomino $P$ and let $p$ be any fixed position in $w$. Let $X$ be the set of all admissible factors overlapping the position $p$ and $\widehat{X}$ be the set of their respective homologue factors. Then, there exist at least one position in $w$ that is not covered by any element of $X \cup \widehat{X}$.

Proof. By contradiction, assume that there is no such point. Let $A \in X$ be the factor that starts at the leftmost position and $B \in X$ be the one that ends at the righmost position as shown below. The homologue factors $A, \widehat{A}$ and $B, \widehat{B}$

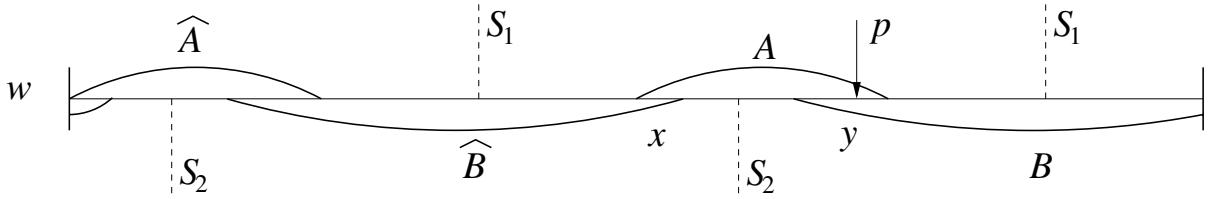

always define two symmetries denoted respectively by $S_{1}$ and $S_{2}$. Let $x$ be the overlap between $A$ and $\widehat{B}$, and $y$ be the overlap between $A$ and $B$. Without loss of generality we may consider that $|y| \geq|x|$. If $|x|=|y|$ the symmetry implies that $x=\widehat{y}$ and the factorization is

$$
w \equiv x U \widehat{x} V x \widehat{U} \widehat{x} \widehat{V} .
$$

We use a property proved in Brlek et all. ( 3], DLT2005) that simplifies a result of Daurat and Nivat [4] on the number of salient and reentrant points of discrete sets: indeed, the number of right turns minus the number of left turns in a closed and non-intersecting path on a square lattice is 4 . In equation 2, notice that all 
turns in a factor are cancelled by those of its homologue. Therefore we only have to consider the turns between consecutive factors. Reading, the word $w$ from left to right, we see that each pair of consecutive factors is cancelled by its homologue: $x U$ is cancelled by $\widehat{U} \widehat{x}, U \widehat{x}$ by $x \widehat{U}, \widehat{x} V$ by $\widehat{V} x$ (the word $w$ is circular), and $V x$ by $\widehat{x} \widehat{V}$. Hence the difference between right and left turns is 0 , and $w$ is self intersecting. Contradiction.

If $|x| \neq|y|$ we have the following situation where the factor $y$ (thick line)

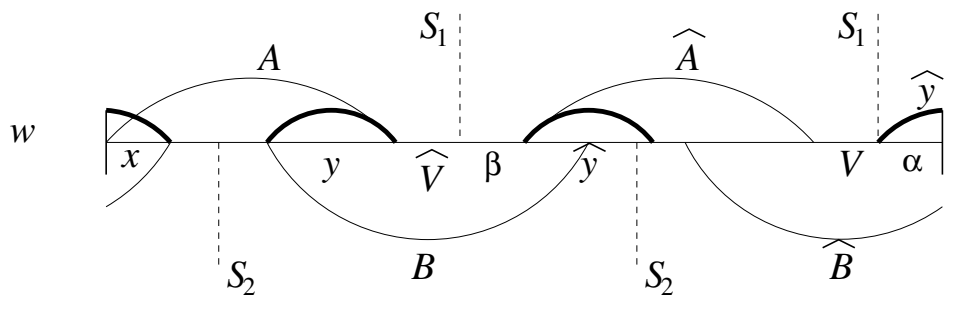

propagates as shown by using the symmetries $S_{1}$ and $S_{2}$. In this case $\widehat{y}$ does not overlap $\widehat{A}$ in $\widehat{B}$, so let $V$ be the factor between $\widehat{A}$ and $\widehat{y}$. We have the following factorization

$$
w \equiv A \widehat{V} \beta \widehat{A} V \alpha .
$$

Passing to vectors, and using commutativity of addition, we have

$$
\vec{w}=\vec{A}+\overrightarrow{\widehat{A}}+\vec{V}+\overrightarrow{\widehat{V}}+\vec{\beta}+\vec{\alpha}=\vec{\beta}+\vec{\alpha}=\overrightarrow{0}
$$

But $\widehat{y}=\alpha x$, so that $\beta$ is followed by $\alpha$ in $w$. Therefore $\beta \alpha$ is a nonempty closed path on the boundary of $P$. Contradiction.

In the case where $\widehat{y}$ does overlap $\widehat{A}$ in $\widehat{B}$ we have the following situation where $\vec{\gamma}+\vec{\beta}=0$ (by closure property $\vec{w}=0=\vec{A}+\vec{\gamma}+\overrightarrow{\widehat{A}}+\vec{\beta}$ ). Moreover,

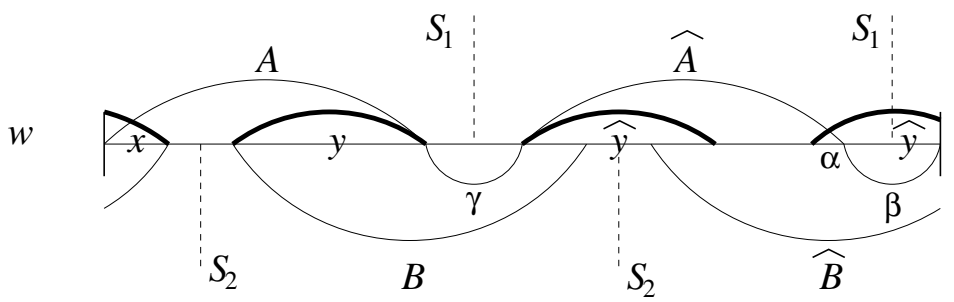

$\widehat{y}=\alpha \beta x$, so that $y \gamma \widehat{y}$ contains the nonempty factor $\widehat{\alpha} \gamma \alpha \beta$ corresponding to a closed path. Contradiction.

Proposition 1 specializes for pseudo-squares as follows. Assume that a pseudosquare $P$ has two factorizations

$$
w=\mathbf{b}(P) \equiv A B \widehat{A} \widehat{B} \equiv X Y \widehat{X} \widehat{Y}
$$

where $A=s X t$. Then, by using the same argument as in the proof above, the boundary of $P$ contains a loop yielding a contradiction. 
Corollary 1. If $w=\mathbf{b}(P) \equiv A B \widehat{A} \widehat{B} \equiv X Y \widehat{X} \widehat{Y}$ are two distinct factorizations of the boundary of a pseudo-square $P$, then there exist $\alpha, \beta, \gamma$ such that $A=\alpha \beta$ and $X=\beta \gamma$.

As an exemple we have the following pseudo-square

$$
a b a \cdot \bar{b} a \bar{b} \cdot \bar{a} \bar{b} \bar{a} \cdot b \bar{a} b \equiv b a b \cdot a \bar{b} a \cdot \bar{b} \bar{a} \bar{b} \cdot \bar{a} b \bar{a}
$$

showing two distinct factorizations. The problem of enumerating all the factorizations of a given pseudo-square will be addressed in a forthcoming paper.

\subsection{A Linear Time Algorithm for Detecting Pseudo-squares}

The main idea used to achieve linear time factorization, is to choose a position $p$ in $w$ and then list all the admissible factors $A$ that overlap this fixed position. The following auxiliary functions are useful. The Longest-Common-Right-Extension (LCRE) and Longest-Common-Left-Extension (LCLE) of two words $u$ and $v$ at positions respectively $m$ and $n$ are partial functions

$$
\text { LCRE, LCLE : } \Sigma^{*} \times \Sigma^{*} \times \mathbb{N} \times \mathbb{N} \longrightarrow \mathbb{N}
$$

defined as follows. For $u, v \in \Sigma^{*}$, let $m$ and $n$ be such that $0 \leq m \leq|u|$ and $0 \leq n \leq|v|$, then

$$
\begin{aligned}
& \operatorname{LCRE}(u, v, m, n)=\operatorname{LCP}\left(\rho^{m}(u), \rho^{n}(v)\right) \\
& \operatorname{LCLE}(u, v, m, n)=\operatorname{LCS}\left(\rho^{|u|-m}(u), \rho^{|v|-n}(v)\right)
\end{aligned}
$$

Remark 1. It is clear from the definition above that LCRE and LCLE may be computed in linear time. Their computation may also be performed directly by the following formulas. Since we use circular words $w$, denote $\underline{m}=m \bmod |w|$. If $u[\underline{m}]=v[\underline{n}]$ then

(i) $\operatorname{LCRE}(u, v, \underline{m}, \underline{n})=\max \{k \in \mathbb{N} \mid u[\underline{m} . .(\underline{m}+\underline{k})]=v[\underline{n} . .(\underline{n}+\underline{k})]\}+1$,

(ii) $\operatorname{LCLE}(u, v, \underline{m}, \underline{n})=\max \{k \in \mathbb{N} \mid u[(\underline{m}-\underline{k}) . . \underline{m}]=v[(\underline{n}-\underline{k}) . . \underline{n}]\}+1$,

and, otherwise, $\operatorname{LCRE}(u, v, \underline{m}, \underline{n})=\operatorname{LCLE}(u, v, \underline{m}, \underline{n})=0$.

For example, if $u=$ aabbbaabaababababa, $v=$ babaabbbaabbabababb, $i=4$ and $j=7$ then (note that the words all starts at position 0) we have

$$
\begin{aligned}
& u=\underline{a a b b} \mathbf{b} \underline{a a b} a a b a b a b a b a, \\
& v=b a b \underline{a a b b} \mathbf{b} \underline{a a b} b a b a b a b b,
\end{aligned}
$$

and $\operatorname{LCRE}(u, v, 4,7)=4, \operatorname{LCLE}(u, v, 4,7)=5$. On the other hand $\operatorname{LCRE}(\mathrm{u}, \mathrm{v}, 4,1)$ $=\operatorname{LCLE}(\mathrm{u}, \mathrm{v}, 4,1)=0$.

Later we will need to perform these computations $\mathcal{O}(n)$ times. Fortunately, the computation of LCLE and LCRE is achieved in constant time, provided a linear preprocessing is performed on $u$ and $v$, by a clever utilization of suffix trees. 
Indeed, for computing $\operatorname{LCRE}(u, v, i, j)$, one proceeds by building a common suffix tree for both $u$ and $v$, and a second pass consists in labeling each node in such a way that the least common ancestor of two nodes is computed in constant time. Both building the suffix tree and its preprocessing is performed in $\mathcal{O}(n)$. For a more detailed description see Gusfield [9], section 9.1, or Gusfield and Stoye [8], page 531 .

For computing $\operatorname{LCLE}(u, v, i, j)$, it suffices to process the mirror images and compute $\operatorname{LCRE}(\widetilde{u}, \widetilde{v},|u|-i-1,|v|-j-1)$.

Lemma 2. Let $w=\mathbf{b}(P)$ be the boundary of $P$. For each occurrence of $A$ in $w$ and each occurrence of $A$ in $\widehat{w}$, whether $A$ is admissible or not is decidable in constant time.

Proof. Given an occurrence of $A$ in $\widehat{w}$, one computes in constant time the corresponding position of $\widehat{A}$ in $w$. If $\widehat{A}$ overlaps $A$ in $w$ is decidable in constant time. If $\widehat{A}$ and $A$ do not overlap then, $u \equiv A x \widehat{A} y$ and $A$ is an admissible factor, by definition, if and only if the three following conditions are verified : $|x|=|y|$, $x_{0} \neq \overline{x_{k-1}}$ and $y_{0} \neq \overline{y_{k-1}}$ where $k=|x|=|y|$.

Lemma 3. Let $w=\mathbf{b}(P) \in \Sigma^{2 n}$ be the boundary of $P$. For any position $p$ in $w$, listing all the admissible factors overlapping $p$ is computed in linear time.

Proof. The following algorithm list all admissible factors containing the $p$-th letter $w$. Since the longest common right and left extension problem can be solved in constant time after linear time preprocessing.

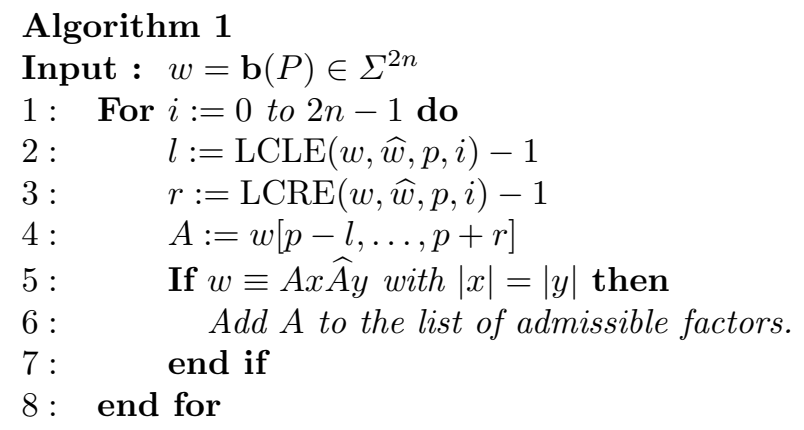

Using the modulo in managing the positions is superfluous because we may assume, without loss of generality (since $w$ is a circular word) that $p=n$. Note that, by definition of LCRE and LCLE, the factor $A$ in this algorithm is necessarily saturated. As shown in Lemma 2, the condition can be tested in constant time by direct computation of positions in $w$. Finally, the loop is performed exactly $2 n$ times.

Remark 2. This lemma implies that the number of admissible factors in a word is linear. To determine a precise upper bound remains an open problem which is similar to the problem of determining a tight upper bound for the number of distinct squares in a word (see for instance Lothaire [11] or Ilie [10]). 
Theorem 1. Let $w=\mathbf{b}(P) \in \Sigma^{2 n}$ be the boundary of P. Determining if $w$ codes a pseudo-square is decidable in linear time.

Proof. If $w$ encodes an exact polyomino, any position belongs to some admissible factor of the Beauquier-Nivat factorization. Therefore, it suffices to apply Lemma 3 to an arbitrary position $p$. Then, Algorithm 1 provides the list of all admissible factors overlapping the position $p$, and it only remains to check, for each admissible factor, if $x=\widehat{y}$. Lemma 1 ensures that if $w \equiv A B \widehat{A} \widehat{B}$ then $B$ is saturated, as shown below.

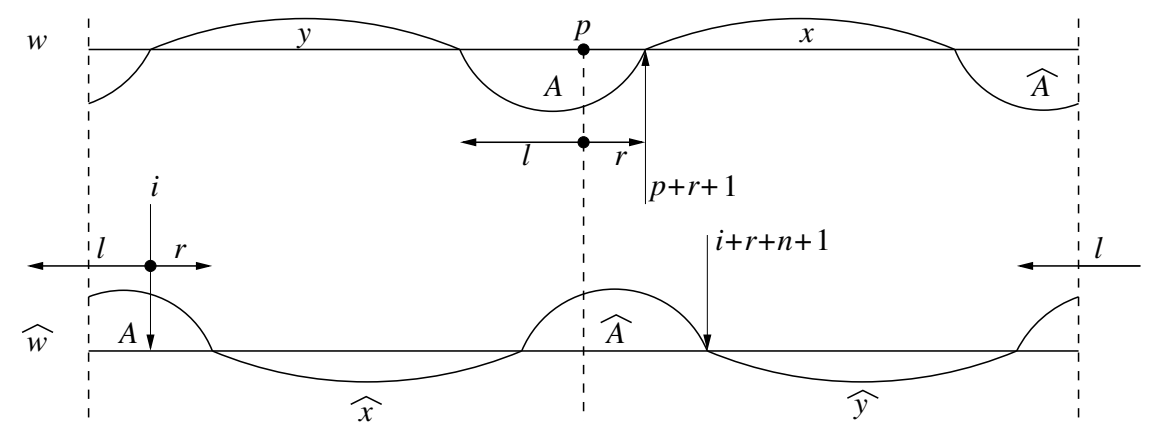

It suffices now to replace step 6 in Algorithm 1 by:

6a: If $\operatorname{LCRE}(w, \widehat{w}, p+r+1, \underline{i+r+n+1})=|x|$ then

$6 b: \quad P$ is a pseudo-square.

$6 c:$ End if

Since LCRE is computed in constant time, the overall algorithm is linear.

\section{Concluding Remarks}

Using combinatorics on words as a tool for analyzing shapes proved powerful as shown in a recent paper by Brlek et al. 3], where an elementary proof of a result of Daurat and Nivat [4] establishing a relation between convex and concave points in discrete figures. In addition, this approach is algorithmic and brings some new insight for addressing geometrical problems.

The results presented here can be extended to more general tilings. Indeed, since the Beauquier-Nivat factorization involves path properties, there is no need for a tile to by a polyomino. For instance, the tile $T$ below might be also called pseudo-square and its Beauquier-Nivat factorization (starting from $\mathbf{S}$ ) is

$$
\mathbf{b}(T)=a a a a b \bar{a} b a a \bar{b} \bar{a} \bar{b} a \bar{b} a \cdot b a b b a b \cdot \bar{a} b \bar{a} b a b \bar{a} \bar{a} \bar{b} a \bar{b} \bar{a} \bar{a} \bar{a} \bar{a} \cdot \bar{b} \bar{a} \bar{b} \bar{b} \bar{a} \bar{b} .
$$

In another direction, it is quite natural to extend the method for pseudohexagons. The situation reveals more complicated, and we designed an optimal algorithm for detecting pseudo-hexagons for a restricted class of closed paths, namely those not having too large squares. This will be described in an extended 

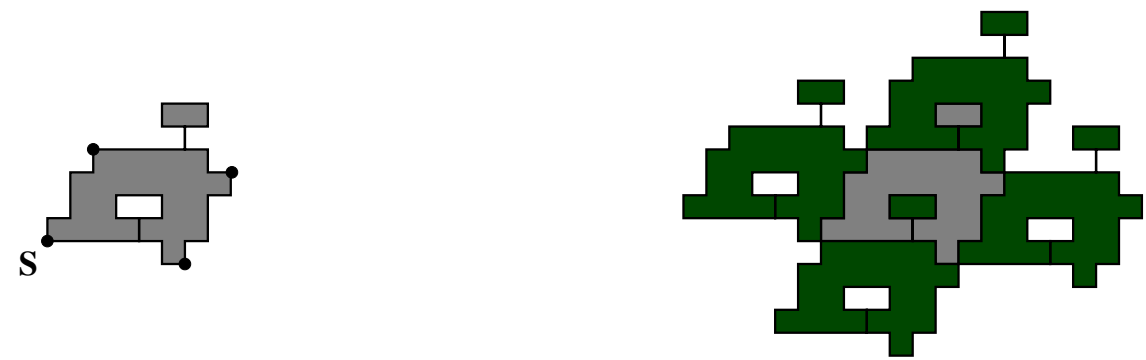

version of the present paper, hoping to lift the condition in order to provide an optimal algorithm without restrictions.

Acknowledgements. The authors are grateful to the anonymous referees for their careful reading and valuable comments.

\section{References}

1. Braquelaire, J.-P., Vialard, A.: Euclidean Paths: A New Representation of Boundary of Discrete Regions, Graphical Models and Image Processing61(1) (1999) 16-43

2. Beauquier, D., Nivat, M.: On Translating one Polyomino to Tile the Plane, Discrete Comput. Geom. 6 (1991) 575-592

3. Brlek, S., Labelle, G., Lacasse, A.: A note on a result of Daurat and Nivat, in C. de Felice and A. Restivo (Eds.) Proc. DLT 2005, 9-th International Conference on Developments in Language Theory (Palermo, Italia, July 4-8, 2005) SpringerVerlag LNCS 3572, 189-198

4. Daurat, A., Nivat, M.: Salient and Reentrant Points of Discrete Sets, In Del Lungo, A., Di Gesu, V., Kuba, A., (Eds.) Proc. International Workshop on Combinatorial Image Analysis (IWCIA'03) (Palermo, Italy, 14-16 May 2003), Electronic Notes in Discrete Mathematics 12, Elsevier Science Publishers (2003)

5. Freeman, H.: On the Encoding of Arbitrary Geometric Configurations, IRE Trans. Electronic Computer 10 (1961) 260-268

6. Freeman, H.: Boundary encoding and processing, in B.S. Lipkin and A. Rosenfeld (Eds.) Picture Processing and Psychopictorics, (Academic Press, New York, 1970) 241-266

7. Gambini, I., Vuillon, L. : An algorithm for deciding if a polyomino tiles the plane by translations, LAMA research report (2003)

8. Gusfield, D., Stoye, J.: Linear time algorithms for finding and representing all the tandem repeats in a string, Journal of Computer and System Sciences 69 (2004) $525-546$

9. Gusfield, D. : Algorithms on Strings, Trees and Sequences, Cambridge University Press, Cambridge (UK) 1997

10. Ilie, L.: A note on the number of distinct squares in a word, In S. Brlek and C. Reutenauer (Eds.) Proc. 5th International Conference ion Words, Words2005 (Montreal, Canada, 13-17 September 2005) Publications du LaCIM 36 (2005) 289-294

11. Lothaire, M.: Applied Combinatorics on words, Cambridge University Press, Cambridge (UK) 2005

12. Wijshoff, H.A.G., Van Leeuven, J.: Arbitrary versus periodic storage schemes and tesselations of the plane using one type of polyomino, Inform. Control 62 (1984) 1-25 